\title{
Fas est impliqué dans l'apoptose induite par les agents anticancéreux indépendamment de toute interaction avec son ligand
}

La mort cellulaire induite par les agents cytotoxiques utilisés en chimiothérapie est le plus souvent une mort par apoptose. Au niveau moléculaire, l'étape finale de l'apoptose implique habituellement la libération, par la mitochondrie, de molécules comme le cytochrome c et la protéase AIF (apoptosis inducing factor) $(m / s$ 1999, n०3, p. 436) sous le contrôle des protéines de la famille Bcl-2. Dans le cytosol, le cytochrome c, en présence d'ATP et de la protéine APAF-1, active la caspase-9 qui, à son tour, active la caspase-3, élément central du processus apoptotique dans de nombreux systèmes cellulaires [1]. En revanche, on connaît beaucoup moins bien les étapes qui, en amont de cette cascade apoptotique, sont mises en jeu lors de l'action d'un agent cytotoxique. La molécule Fas (APO-1/CD95) pourrait-elle être un intermédiaire important ?

Fas est un récepteur transmembranaire qui comporte des «domaines de mort» (death domain, DD), indispensables à l'induction de la mort cellulaire. Le ligand de ce récepteur (FasL/APO-1L/CD95L) est une protéine membranaire de la famille du $\mathrm{TNF} \alpha$ (tumor necrosis factor $\alpha$ ) dont le domaine carboxy-terminal est extracellulaire. La fixation de FasL sur Fas entraîne l'agrégation des récepteurs Fas $\left(m / s ~ 1998, n^{\circ} 4, p .511\right)$, réaction qui fait intervenir le «domaine de mort». Ce domaine interagit avec des protéines «adaptatrices», dont FADD (Fas associated death domain) qui joue un rôle-clé dans la transduction d'un signal de mort [2]. FADD recrute la procaspase-8 dont l'activation conduit à l'apoptose, soit en favorisant la libération de molécules pro-apoptotiques par la mitochondrie, soit en activant directement les caspases effectrices [3] (figure 1).
Comment Fas pourrait-il intervenir dans l'apoptose provoquée par les agents cytotoxiques? On sait que l'expression de FasL à la surface de certaines cellules tumorales est induite par les drogues cytotoxiques, et ce par un mécanisme qui ferait intervenir la production de céramide et la protéine p53. Il était logique de formuler l'hypothèse selon laquelle l'interaction autocrine ou paracrine de FasL avec son récepteur Fas participait à l'apoptose induite par les agents anticancéreux. Cette hypothèse reposait sur l'observation que des fragments $\left.\mathrm{F}(\mathrm{ab})^{\prime}\right)_{2}$ d'un anticorps anti-APO-1 inhibaient l'apoptose des cellules leucémiques CEM traitées par la doxorubicine [4]. La controverse est née de la constatation que la fixation sur Fas-L de l'anticorps antagoniste ZB4, ou de la molécule de fusion Fas-IgG, ne prévenait l'apoptose ni des cellules leucémiques CEM et U937 ni des cellules cancéreuses coliques HT29 et HCT116 exposées à divers agents cytotoxiques [5]. Or l'anticorps ZB4 et la molécule FasIgG sont pourtant capables de bloquer l'induction de l'apoptose par le ligand de Fas ou l'anticorps agoniste CH11. Fas, si tant est qu'il ait été impliqué dans l'apoptose induite par les agents cytotoxiques, ne l'était pas par sa liaison à FasL.

Une première indication est venue des données de microscopie confocale: nous avons en effet observé que des molécules telles que le cisplatine, l'étoposide ou la vinblastine pouvaient induire l'agrégation des récepteurs Fas à la surface de lignées leucémiques et de lignées cancéreuses coliques [5]. Cette agrégation est observée en l'absence d'expression de FasL à la surface des cellules tumorales. Il en est de même pour les hépatocytes exposés aux sels biliaires [6]. L'agrégation de Fas induit le recrutement de la protéine FADD dont le niveau d'expression rend compte de la sensibilité des cellules tumorales à l'apoptose induite par les agents cytotoxiques. En effet, la surexpression de FADD sensibilise les cellules cancéreuses coliques HT29 à l'effet cytotoxique du cisplatine. On observe une diminution de la sensibilité des cellules tumorales à divers agents anticancéreux lorsque FADD est neutralisé par la transfection transitoire d'une construction codant soit pour un ARN antisens de FADD, soit pour un mutant dominant négatif de FADD, soit pour des protéines virales (MC159 du virus du molluscum contagiosum ou E8 du virus herpès équin) qui s'opposent à l'interaction de FADD avec la procaspase-8 [5]. Nous avons conclu de ces observations que Fas pouvait jouer un rôle dans la réponse apoptotique aux médicaments anticancéreux, indépendamment de toute interaction avec son ligand.

L'expression de Fas à la surface des cellules malignes diminue avec la progression tumorale et/ou avec l'acquisition d'un phénotype de résistance aux agents cytotoxiques. L'expression de Fas à la surface de certaines cellules tumorales peut être restaurée ou accrue par des drogues anticancéreuses ayant des mécanismes d'action très variés (cisplatine, doxorubicine, campthothécine, 5-fluoro-uracile, mitomycine C) [7]. Ces mêmes drogues induisent aussi l'accumulation de molécules pro-apoptotiques telles que FADD, les procaspases-2, -3 et -8, et Bax. L'accumulation de Fas et de ces molécules pro-apoptotiques implique, au moins en partie, un mécanisme transcriptionnel $[8,9]$. Notre hypothèse est que l'augmentation de l'expression de Fas et de 


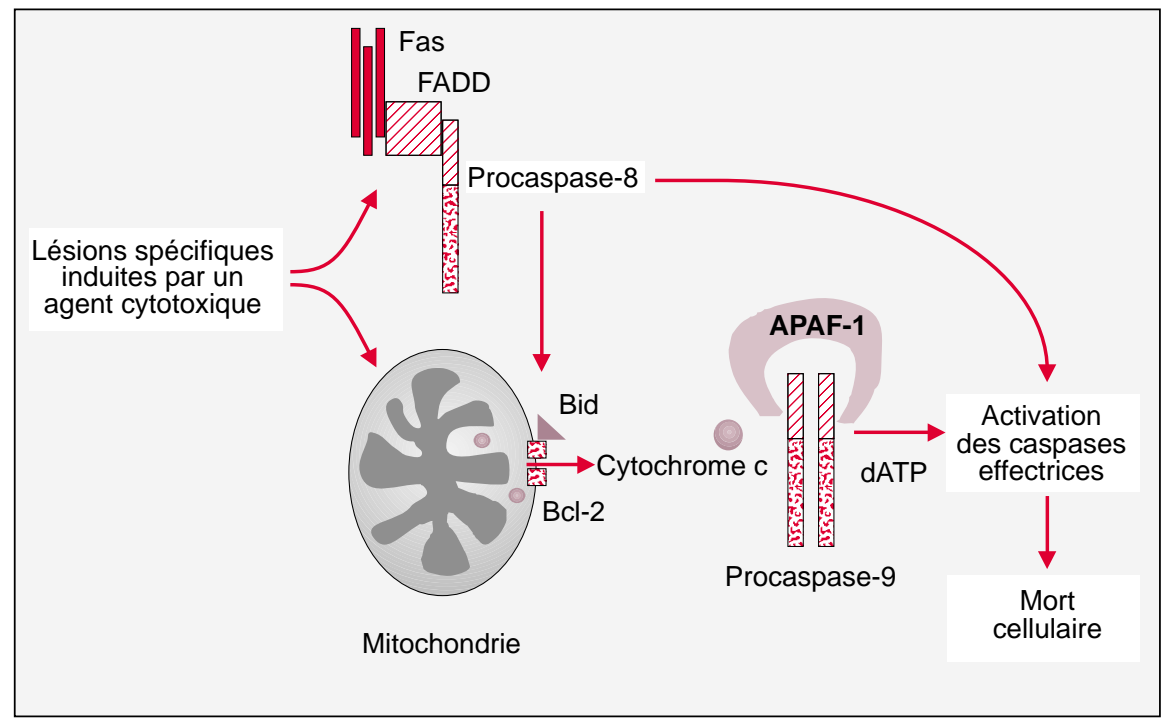

Figure 1. Induction de I'apoptose cellulaire par les agents anticancéreux. Les agents anticancéreux peuvent induire l'apoptose par deux voies complémentaires: I'une agit directement sur la mitochondrie, et l'autre passe par les récepteurs Fas mais ne requiert pas la liaison du ligand de Fas.

FADD favoriserait l'agrégation du récepteur, le recrutement de la protéine adaptatrice et l'activation de procaspases telles que la procaspase8. La caspase-8 pourrait alors cliver la molécule Bid [10], favorisant la libération des molécules pro-apoptotiques localisées dans la mitochondrie, ou activer directement les caspases effectrices (figure 1).

Fas et FADD ne sont sans doute pas indispensables à l'activité cytotoxique des médicaments anticancéreux mais ils peuvent y contribuer. Ainsi, l'augmentation de l'expression de FADD à l'aide de vecteurs rétroviraux sensibilise les cellules tumorales à la chimiothérapie in vitro et in vivo dans des modèles de gliomes malins humains et murins [11]. Fas n'est pas le seul récepteur à domaine de mort

\author{
Marie-Thérèse Dimanche-Boitrel \\ Olivier Micheau \\ Éric Solary
}

Inserm U. 517, Facultés de médecine et de pharmacie, 7, boulevard Jeanne-d'Arc, 21000 Dijon, France.

1. Mignon A, Rouquet N, Joulin V. Les caspases, les protéases à cystéine de l'apoptose : un enjeu thérapeutique pour demain ? Med Sci 1998; 14: 9-17.

2. Zhang JK, Cado D, Chen A, Kabra NH, Winoto A. Fas-mediated apoptosis and activation-induced T-cell proliferation are defective in mice lacking FADD/Mort1. Nature 1998; 392: 296-300.

3. Scaffidi C, Fulda S, Srinivasan A, et al. Two CD95 (APO-1/Fas) signaling pathways. EMBO J 1998; 17 : 1675-87.

4. Friesen C, Herr I, Krammer PH, Debatin KM. Involvement of the CD95 (APO-1/Fas) system in drug-induced apoptosis in leukemia cells. Nat Med 1996; 2 : 574-7.

5. Micheau O, Solary E, Hammann A, DimancheBoitrel MT. Fas-ligand-independent FADD-mediated activation of the Fas death pathway by anticancer drugs. J Biol Chem 1999; 274: 7987-92.

6. Faubion WA, Guicciardi ME, Miyoshi H, et al. Toxic bile salts induce rodent hepatocyte apoptosis via direct activation of Fas. J Clin Invest 1999; 103: $137-45$.

7. Micheau O, Solary E, Hammann A, DimancheBoitrel MT. Sensitization of cancer cells treated with cytotoxic drugs to Fas-mediated cytotoxicity. J Natl Cancer Inst 1997; 89: 783-9.

8. Micheau O, Hammann A, Solary E, DimancheBoitrel MT. STAT-1 independent upregulation of Fas (CD95/APO-1), FADD and procaspases in cancer cells treated with cytotoxic drugs. Biochem Biophys Res Commun 1999; 256: 603-7.

9. Droin N, Dubrez L, Eymin B, et al. Upregulation of CASP genes in human tumor cells undergoing etoposide-induced apoptosis. Oncogene 1998 ; 16 : 2885-94.

10. Li H, Zhu H, Xu CJ, Yuan J. Cleavage of BID by caspase 8 mediates the mitochondrial damage in the Fas pathway of apoptosis. Cell 1998; 94: 491-501. 11. Kondo S, Ishizaka Y, Okada T, et al. FADD gene therapy for malignant gliomas in vitro and in vivo. Hum Gen Ther 1998; 9: 1599-608.

12. Walczak H, Miller RE, Ariail K, et al. Tumoricidal activity of tumor necrosis factor-related apoptosis-inducing ligand in vivo. Nat Med 1999; 5 : 157-63. 